Article

\title{
Inflammatory Proteins HMGA2 and PRTN3 as Drivers of Vulvar Squamous Cell Carcinoma Progression
}

\author{
Agnieszka Fatalska ${ }^{1,+}{ }^{+}$, Natalia Rusetska ${ }^{2,+}{ }^{,}$Elwira Bakuła-Zalewska ${ }^{3}$, Artur Kowalik ${ }^{4,5}$ (D, Sebastian Zięba ${ }^{4}$, \\ Agnieszka Wroblewska ${ }^{6}$, Kamil Zalewski ${ }^{2,7,8}$, Krzysztof Goryca 9,10, Dominik Domański ${ }^{1}$ (D) \\ and Magdalena Kowalewska 2,11,*(D)
}

1 Mass Spectrometry Laboratory, Institute of Biochemistry and Biophysics-Polish Academy of Sciences, 02-106 Warsaw, Poland; fataga@wp.pl (A.F.); dom.domanski@ibb.waw.pl (D.D.)

2 Department of Molecular and Translational Oncology, Maria Sklodowska-Curie National Research Institute of Oncology, 02-781 Warsaw, Poland; natarusetska@gmail.com (N.R.); zalewski81@gmail.com (K.Z.)

3 Department of Pathology, Maria Sklodowska-Curie National Research Institute of Oncology, 02-781 Warsaw, Poland; elwirabz@onet.eu

4 Department of Molecular Diagnostics, Holycross Cancer Center, 25-734 Kielce, Poland; arturko@onkol.kielce.pl (A.K.); s.zieba@o2.pl (S.Z.)

5 Division of Medical Biology, Institute of Biology, Jan Kochanowski University, 25-369 Kielce, Poland

6 Department of Gynecology, Holy Family Hospital, 02-544 Warsaw, Poland; agnwr@op.pl

7 Department of Gynecologic Oncology, Holycross Cancer Center, 25-734 Kielce, Poland

8 Chair and Department of Obstetrics, Gynecology and Oncology, 2nd Faculty of Medicine, Warsaw Medical University, 03-242 Warsaw, Poland

9 Department of Genetics, Maria Sklodowska-Curie National Research Institute of Oncology, 02-781 Warsaw, Poland; kgoryca@gmail.com

10 Genomics Core Facility, Centre of New Technologies, University of Warsaw, 02-097 Warsaw, Poland

11 Department of Immunology, Biochemistry and Nutrition, Centre for Preclinical Research and Technologies, Medical University of Warsaw, 02-097 Warsaw, Poland

* Correspondence: magdalena.kowalewska@pib-nio.pl; Tel.: +48-22-5462650

+ These authors contributed equally to this work.

Citation: Fatalska, A.; Rusetska, N.; Bakuła-Zalewska, E.; Kowalik, A.; Zięba, S.; Wroblewska, A.; Zalewski, K.; Goryca, K.; Domański, D.; Kowalewska, M. Inflammatory Proteins HMGA2 and PRTN3 as Drivers of Vulvar Squamous Cell Carcinoma Progression. Cancers 2021, 13, 27. https://dx.doi.org/10.3390/ cancers13010027

Received: 10 November 2020 Accepted: 18 December 2020 Published: 23 December 2020

Publisher's Note: MDPI stays neutral with regard to jurisdictional claims in published maps and institutional affiliations.

Copyright: () 2020 by the authors. Licensee MDPI, Basel, Switzerland. This article is an open access article distributed under the terms and conditions of the Creative Commons Attribution (CC BY) license (https: / / creativecommons.org/ licenses/by/4.0/).
Simple Summary: Our study aimed to advance the understanding of vulvar squamous cell carcinoma (VSCC) biology by recognizing biological pathways that drive the progression of this disease. We applied the experimental path from global proteomic analysis of vulvar tumors to the targeted and quantitative assessment of specific proteins both in the tumors and blood of VSCC patients. The proteomic analysis has advanced the knowledge on VSCC biology by pointing at inflammation as a driver of progression and by providing grounds for the hypothesis of vulvovaginal microflora disturbances as a trigger for the inflammatory response. The study results indicate prognostic protein markers and potential therapeutic targets for improved and personalized management of VSCC.

Abstract: Current knowledge on the biology of squamous cell vulvar carcinoma (VSCC) is limited. We aimed to identify protein markers of VSCC tumors that would permit to stratify patients by progression risk. Early-stage tumors from patients who progressed (progVSCC) and from those who were disease-free (d-fVSCC) during follow-up, along with normal vulvar tissues were examined by mass spectrometrybased proteomics. Differentially expressed proteins (DEPs) were then verified in solid tissues and blood samples of patients with VSCC tumors and vulvar premalignant lesions. In progVSCC vs. d-fVSCC tumors, the immune response was the most over-represented Gene Ontology category for the identified DEPs. Pathway profiling suggested bacterial infections to be linked to aggressive VSCC phenotypes. High Mobility Group AT-Hook 2 (HMGA2) and Proteinase 3 (PRTN3) were revealed as proteins predicting VSCC progression. HMGA2 and PRTN3 abundances are associated with an aggressive phenotype, and hold promise as markers for VSCC patient stratification. It appears that vulvovaginal microflora disturbances trigger an inflammatory response contributing to cancer progression, suggesting that bacterial rather than viral infection status should be considered in the development of targeted therapies in VSCC.

Keywords: HMGA2; iTRAQ; microbiome; proteomics; PRTN3; vulvar carcinoma 


\section{Introduction}

Vulvar carcinoma is a rare genital malignancy contributing to the estimated 44,235 new cases and 15,222 cancer-related deaths in 2018 [1]. Surgical management is at the cornerstone of treatment for most vulvar cancers, while radiotherapy is added in advanced cases. Vulvar carcinoma spreads primarily by local expansion and the lymphatic system. The status of the regional lymph nodes (LN) is the most important prognostic factor for vulvar carcinoma patients and therefore it is included in the International Federation of Gynecology and Obstetrics and American Joint Committee on Cancer TNM staging systems for vulva. The five-year overall survival rates are approximately $85.5 \%$ and $50.6 \%$ in patients without and with LN involvement, respectively [2]. However, the LN status can only be determined by histological examination of the resected LNs, i.e., lymphadenectomy or sentinel LN biopsy, which are performed in patients eligible for surgery [3]. Thus, these invasive and often highly morbid surgeries cannot be avoided as their benefits outweigh the risk of not detecting the cancer spread in the regional LNs and thus the risk of relapse. Newer strategies, combining surgery, radio- and chemotherapy, and tailoring the treatment to the extent of clinical and pathologic disease, are used in advanced and metastatic disease. Patterns of practice in combining these treatments vary $[4,5]$.

Ninety percent of vulvar cancers are squamous cell carcinomas (VSCC), which arise via high-risk human papilloma virus (hrHPV)-dependent and -independent pathways. The first pathway begins with the formation of high-grade squamous intraepithelial lesions (HSIL), while the other with a differentiated-type vulvar intraepithelial neoplasia (dVIN) [6]. Although p16-positivity considered a surrogate for hrHPV-positive cases is related with better survival [7], so far, there is no difference in therapeutic options for hrHPV-dependent and -independent disease [8]. Our recent review [9] of next-generation sequencing studies allowed recognition of the phosphatidylinositol 3-kinase (PI3K)/ protein kinase B (AKT) signaling pathway as the main pathway deregulated in both hrHPV-induced and hrHPVunrelated VSCC. Thus, novel therapeutic options targeting the PI3K/Akt pathway should be considered as potentially effective in VSCC patients' management. The availability of biomarkers predictive for tumor aggressiveness would assist surgical decisions and enable individualized treatment. Unfortunately, clinically relevant, surgery-independent markers are still lacking for VSCC patients.

Extracting information on tumor-driving molecular changes in order to predict tumor development is one of the biggest challenges for translational oncology. The aim of this study was to advance the understanding of the VSCC biology by recognizing biological pathways that drive VSCC progression. For the analysis of protein expression, isobaric tags for relative and absolute quantitation (iTRAQ), a global mass spectrometry-based proteomic method was employed, which has so far proved successful in discovery-type experiments in identifying diagnostic cancer biomarkers [10]. Proteomic profiling was performed to characterize VSCC tumors and to identify protein markers that would indicate tumors more likely to progress. In silico investigation of proteomic data was used to provide hypotheses on the mechanism of VSCC progression based on biological pathways' identification. Global techniques, such as iTRAQ, indicate potential biomarker candidates out of thousands of identified proteins. Therefore, consecutive steps of global data validation were applied in this study in search of protein with prognostic value in VSCC.

\section{Results}

\subsection{Identification of Differentially Expressed Proteins Using iTRAQ}

We applied the iTRAQ method to discover proteins with abundance alterations that are characteristic for the aggressive VSCC tumor phenotype, and to identify the molecular pathways underlying VSCC progression. The specimens included 16 VSCC tumor samples of patients that were found disease-free during the follow-up period ("d-fVSCC" group), 12 VSCC tumor samples obtained from patients who progressed ("progVSCC" group) and 14 normal vulvar tissue samples (controls). Among the d-fVSCC samples, eight were 
hrHPV-positive and seven were hrHPV-negative, while among the progVSCC samples, five were hrHPV-positive and seven were hrHPV-negative.

iTRAQ analysis identified 5509 proteins in this set of samples. The thresholds for differential expression was set to a protein level fold-change (FC) of $>1.5$ and $<0.67$, with a $p$-value of $<0.05$. With these thresholds, 586 differentially expressed proteins (DEPs) were identified between VSCCs (both d-fVSCC and progVSCC samples) and controls, of which 206 were increased and 380 were decreased in VSCCs. A set of hundreds of DEPs is difficult to interpret in terms of biological consequences/meaning of functional cellular alterations. Therefore, specialized tools were employed to translate identified DEPs into cellular processes: DEPs were submitted to Gene Ontology (GO) and Kyoto Encyclopedia of Genes and Genomes (KEGG) pathway analyses. GO and KEGG are considered the most comprehensive databases of molecular functions of genes and proteins and associated cellular processes. The results for the top 20 identifiers are provided in Tables S1 and S2, respectively.

We then compared the iTRAQ results obtained for d-fVSCC with those for progVSCC samples. The number of DEPs dropped down to 34, of which 27 were increased and 7 were decreased in levels. The GO and KEGG pathway analyses were conducted for DEPs in d-fVSCC and progVSCC samples. Interestingly, the identified set of significantly enriched GO biological process terms were different from those differentiating VSCCs from controls, and the immune response emerged as the most prevalent (Table S3). KEGG pathway analysis also revealed immune-related pathways among those dysregulated in VSCC (Table S4). DEPs with significant alterations in quantity were also submitted to core analysis in Ingenuity Pathway Analysis (IPA). The 10 canonical pathways most significantly affected in progVSCC compared to d-fVSCC samples (with a 1.2 threshold for the FC value), further emphasized the importance of inflammatory reactions that accompany VSCC progression (Figure 1 and Figure S1).

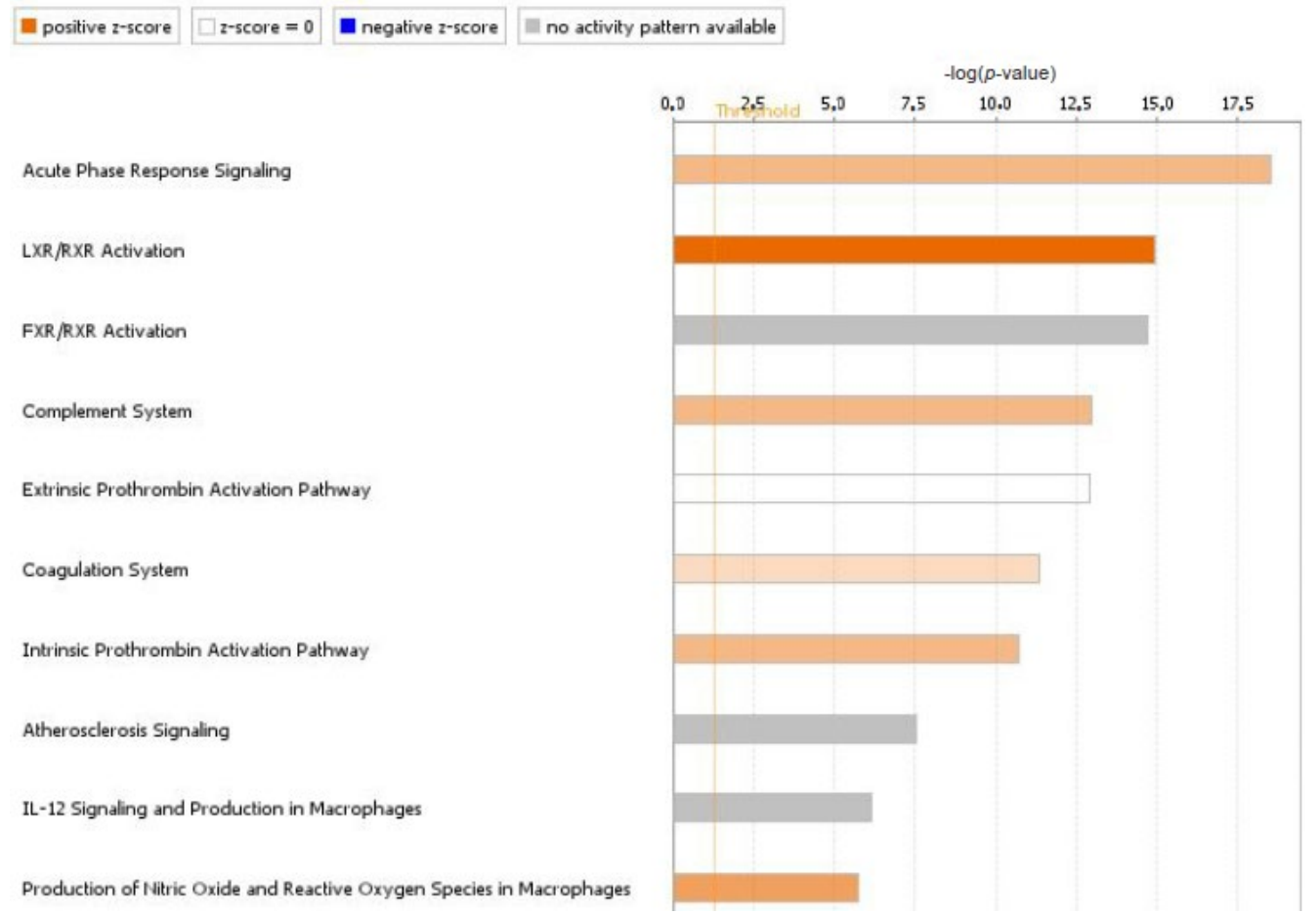

Figure 1. Top 10 canonical pathways enriched in progressed vulvar squamous cell carcinoma (progVSCC) compared to disease-free vulvar squamous cell carcinoma (d-fVSCC) tumors, identified by core analysis in Ingenuity Pathway Analysis (IPA). Top 10 canonical pathways enriched by IPA. The vertical orange line represents the threshold $p$-value (0.05) for pathway's enrichment. The horizontal axis is the -log ( $p$-value). Pathways predicted to be activated are in orange (based on the Z-scores, the activation scores determined by IPA), and grey bars relate to pathways with no activity pattern available. 


\subsection{Verification by Parallel Reaction Monitoring}

To verify our iTRAQ results in a larger sample set, we employed parallel reaction monitoring (PRM) to analyze 49 candidate protein biomarkers of VSCC (Table S5) 23 significantly differentially expressed proteins differentiated d-fVSCC from progVSCC tumors: 15 were increased and 8 decreased with $\mathrm{FC}>1.5$ and $<0.9$, respectively. Areas under receiver operating characteristics (ROC) curves (AUC) served to assess the feasibility of individual DEPs in distinguishing between d-fVSCC and progVSCC samples and between VSCC and normal vulvar tissues (Table 1).

Table 1. The list of 23 differentially expressed proteins (DEPs) identified in the iTRAQ experiment and confirmed using PRM analysis.

\begin{tabular}{ccccccccc}
\hline \multirow{2}{*}{ Protein } & \multicolumn{3}{c}{ d-fVSCC vs. progVSCC } & \multicolumn{5}{c}{ VSCC vs. Normal Vulvar Tissues } \\
\cline { 2 - 8 } & $\boldsymbol{p}$-Value & adjp & FC & AUC & $p$-Value & adjp & FC & AUC \\
\hline HMGA2 & 0.0063 & 0.0824 & 14.4 & 0.73 & 0.0059 & 0.0095 & 45.5 & 0.76 \\
ANO1 & 0.0068 & 0.0824 & 3.37 & 0.73 & 0.6177 & 0.6312 & 1.80 & 0.55 \\
PRTN3 & 0.0117 & 0.0981 & 2.75 & 0.71 & $<0.0001$ & $<0.0001$ & 13.3 & 0.92 \\
UBE2C & 0.0070 & 0.0824 & 2.72 & 0.73 & 0.0001 & 0.0002 & 8.89 & 0.87 \\
KRT18 & 0.0220 & 0.0981 & 2.48 & 0.70 & $<0.0001$ & 0.0001 & 8.18 & 0.91 \\
S100A12 & 0.0160 & 0.0981 & 2.44 & 0.71 & $<0.0001$ & $<0.0001$ & 12.0 & 0.92 \\
PTX3 & 0.0359 & 0.1211 & 2.31 & 0.68 & 0.0535 & 0.0662 & 2.68 & 0.68 \\
RUNX1 & 0.0192 & 0.0981 & 2.06 & 0.70 & $<0.0001$ & 0.0001 & 6.73 & 0.90 \\
PADI2 & 0.0192 & 0.0981 & 1.89 & 0.70 & $<0.0001$ & $<0.0001$ & 3.72 & 0.88 \\
ISG15 & 0.0273 & 0.1069 & 1.88 & 0.69 & $<0.0001$ & $<0.0001$ & 35.4 & 0.94 \\
ABCC10 & 0.2133 & 0.3992 & 1.75 & 0.61 & 0.0068 & 0.0106 & 5.55 & 0.75 \\
IFIT3 & 0.0811 & 0.1907 & 1.74 & 0.65 & $<0.0001$ & $<0.0001$ & 16.5 & 0.91 \\
IDO1 & 0.8737 & 0.9126 & 1.68 & 0.52 & 0.0001 & 0.0001 & 17.4 & 0.88 \\
PTPMT1 & 0.2067 & 0.3992 & 1.57 & 0.61 & 0.0815 & 0.0957 & 1.72 & 0.67 \\
MX1 & 0.2550 & 0.4279 & 1.50 & 0.60 & $<0.0001$ & $<0.0001$ & 25.5 & 0.93 \\
KRTDAP & 0.6183 & 0.7647 & 0.88 & 0.54 & 0.0034 & 0.0057 & 0.49 & 0.77 \\
OGN & 0.0670 & 0.1852 & 0.81 & 0.66 & $<0.0001$ & $<0.0001$ & 0.20 & 0.91 \\
A2ML1 & 0.1499 & 0.3202 & 0.76 & 0.63 & 0.0329 & 0.0429 & 0.65 & 0.70 \\
EDEM2 & 0.2844 & 0.4435 & 0.74 & 0.59 & 0.1983 & 0.2273 & 1.85 & 0.62 \\
ABI3BP & 0.0230 & 0.0981 & 0.71 & 0.69 & $<0.0001$ & $<0.0001$ & 0.16 & 0.92 \\
PRELP & 0.0160 & 0.0981 & 0.70 & 0.71 & $<0.0001$ & $<0.0001$ & 0.26 & 0.93 \\
CSTA & 0.2925 & 0.4435 & 0.69 & 0.59 & 0.4850 & 0.5066 & 1.12 & 0.57 \\
SPRR3 & 0.0811 & 0.1907 & 0.62 & 0.65 & 0.3964 & 0.4234 & 2.07 & 0.58
\end{tabular}

Abbreviations: adjp, adjusted $p$-value; FC, fold change of differential protein content; AUC, area under receiver operating characteristics (ROC) curves.

\subsection{Immunohistochemistry}

From the dataset showing the list of 23 candidate biomarkers of VSCC progression verified with PRM analysis, two proteins-High Mobility Group AT-Hook 2 (HMGA2) and Proteinase 3 (PRTN3) - were analyzed by immunohistochemistry (IHC) in paraffinembedded tissue specimens obtained from VSCC patients and from those with premalignant vulvar lesions (HSIL and dVIN). HMGA2 and PRTN3 proteins were selected for this analysis based on the difference in their abundance in d-fVSCC vs. progVSCC and in VSCC vs. control samples. Additionally, the AUC values indicated relatively high predictive values of both HMGA2 and PRTN3 to identify VSCC patients at risk of progression.

HMGA2 staining was nuclear or both nuclear and cytoplasmic, and confined to neoplastic cells (Figure 2). In general, HMGA2 staining was weak in premalignant lesions and strong in VSCC tissues. Additionally, significantly higher relative numbers of HMGA2positve neoplastic cells were seen in VSCCs than in vulvar pre-cancers (Figure 2c). The percentages of HMGA2-positve VSCC cells were not statistically significantly different in hrHPV-positive and -negative primary VSCC samples. Survival curves of the two groups of patients with primary VSCC tumors categorized by the percentage of HMGA2-positive cancer cells are shown in Figure 2d. The group with a high number of HMGA2-positive 
cells had a significantly shorter time to progression than patients with a low fraction of HMGA2-positive cells in the tumors. An increase of HMGA2 level may result from an inhibition of let-7 microRNA family members [11]. Our qPCR analysis confirmed a decrease of circulating let-7c levels in plasma of patients with premalignant lesions and VSCC patients compared to healthy volunteers (Figure S2).

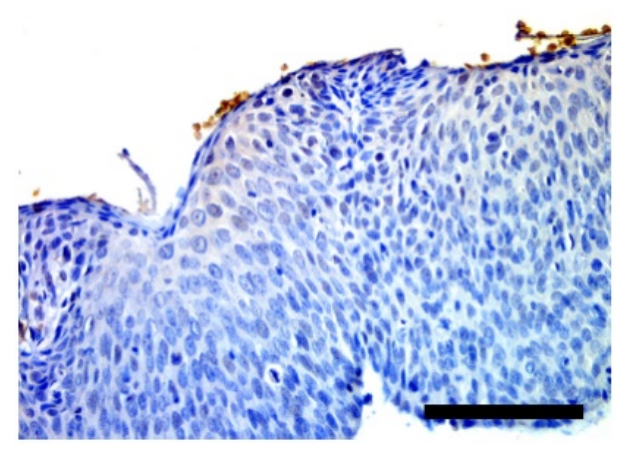

(a)

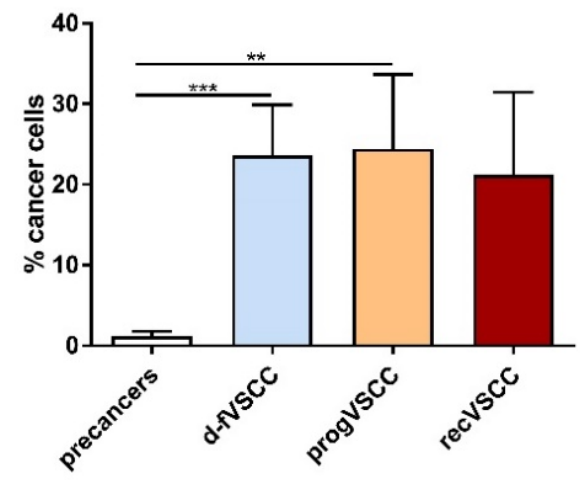

(c)

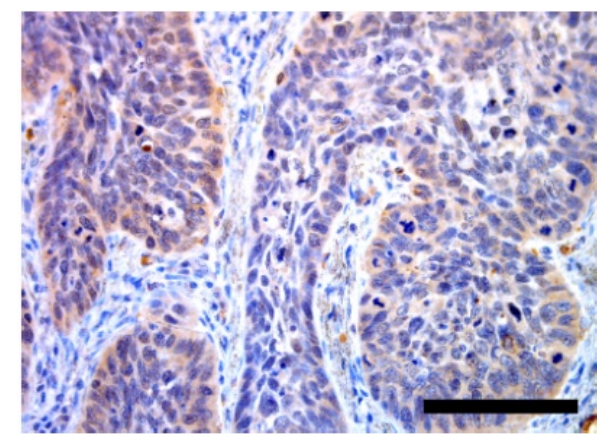

(b)

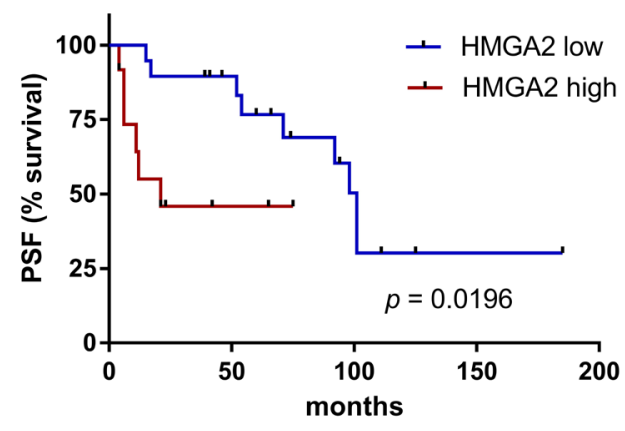

(d)

Figure 2. The percentage of HMGA2-positive neoplastic cells. Examples of immunohistochemical HMGA2 staining performed on tissue sections of high-grade squamous intraepithelial lesions (HSIL) (a) and progVSCC (b) tumor samples. Images were taken at $40 \times$ magnification. Scale bar, $100 \mu \mathrm{m}$. Comparison of the IHC results for premalignant lesions (HSIL; $n=44$ and dVIN; $n=6)$, d-fVSCC $(n=19), \operatorname{progVSCC}(n=13)$ and recVSCC $(n=7)$ samples (c). Survival curve (time to progression) according to the relative numbers of HMGA2-positive cancer cells in primary VSCC tumors $(n=31)(\mathbf{d})$. Significant alternations are indicated by asterisks $\left({ }^{* *}, p\right.$-value $\leq 0.01 ;{ }^{* * *}, p$-value $\left.\leq 0.001\right)$. Abbreviations: PFS, progression-free survival; high and low, high and low proportion of HMGA2-positive cancer cells ( $\geq 30$ and $\leq 10$ cells, respectively).

PRTN3 staining was cytoplasmic and observed in neoplastic cells, macrophages, and lymphocytes (Figure 3). In general, the observed percentage of PRTN3-positive cells was lower among cancer cells than among the infiltrating non-neoplastic cells. Significantly higher relative numbers of PRTN3-positve cells were seen in VSCC than in vulvar premalignant lesions (dVIN and HSIL) (Figure $3 \mathrm{~d}-\mathrm{f}$ ). Survival curves of the two groups of patients with primary VSCC tumors categorized by the number of PRTN3-positive cancer cells are shown in Figure 3c. Patients with higher numbers of PRTN3-positive cancer cells in the tumors had a significantly shorter time to progression than those with low numbers of these cells. The impact of PRTN3-positive macrophage or lymphocyte counts on survival was statistically insignificant. 


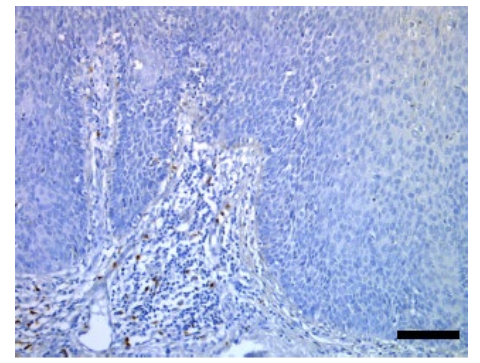

(a)

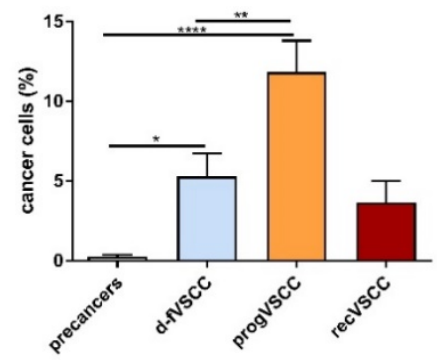

(d)

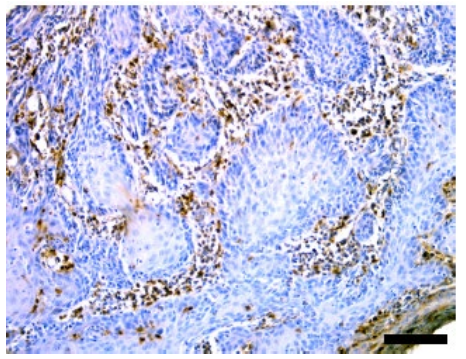

(b)

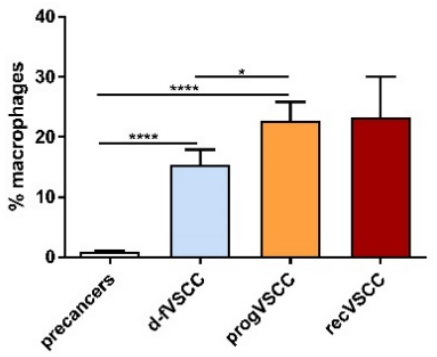

(e)

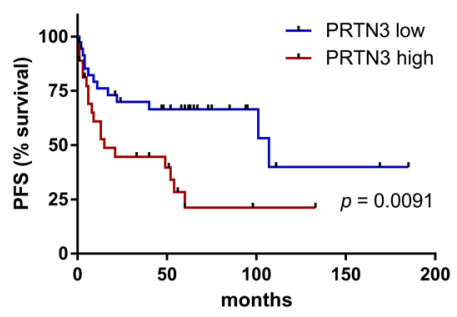

(c)

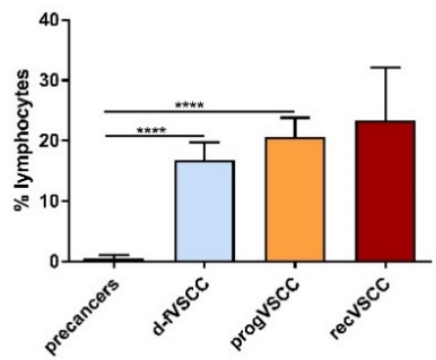

(f)

Figure 3. The results of PRTN3 immunostaining. Examples of immunohistochemical PRTN3 staining performed on tissue sections of HSIL (a) and VSCC tumors (b). Images were taken at 20x (a-b). Scale bar, $100 \mu \mathrm{m}$. The percent of PRTN3-positive neoplastic cells (d), macrophages (e), and lymphocytes (f) in vulvar pre-cancers (HSIL; $n=40$ and dVIN; $n=5)$, d-fVSCC $(n=34), \operatorname{progVSCC}(n=31)$ and recurrent VSCC $(n=3)$ samples. Survival curve (time to progression) according to the proportion of PRTN3-positive cancer cells (c) in primary VSCC tumors $(n=64)$. Significant alternations are indicated by asterisks ${ }^{*}, p$-value $\leq 0.05 ;{ }^{* *}, p$-value $\leq 0.01 ;{ }^{* * *}, p$-value $\leq 0.001 ;{ }^{* * * *}, p$-value $\left.\leq 0.0001\right)$. Abbreviations: PFS, progression-free survival; high and low, high and low proportions of PRTN3-positive cells.

\subsection{PRTN3 Screening in VSCC Patients' Blood}

PRTN3, secreted by neutrophils [12,13], belongs to neutrophil serine proteases, enzymes connecting innate immunity and coagulation [14]. Indeed, the complement and coagulation cascades pathway was at the top of the KEGG pathways identified in comparison of d-fVSCC and progVSCC samples by iTRAQ (Table S4, Figure S3). Therefore, to determine whether systemic alterations follow the phenomena observed in the analyzed tissues, the presence of PRTN3 was assessed by ELISA in the serum samples of patients with vulvar premalignant lesions and in VSCC patients, as well as in healthy women. The results of the measurement of PRTN3 as well as of anti-PRTN3 antibodies (ANCA, anti-neutrophil cytoplasmic antibodies) are shown in Figure 4a,b. Survival curves of the two groups of patients with primary VSCC tumors categorized by the PRTN3 and ANCA serum levels are shown in Figure $4 \mathrm{c}, \mathrm{d}$, respectively. The group of patients with primary VSCC with higher PRTN3 levels had a significantly shorter time to progression than the low PRTN3 group (15 vs. 57 months). The survival curves were not significantly different for patients with primary VSCC categorized by ANCA serum levels.

PRTN3 [14] is a modulator of human platelets' function [15]. Platelets amplify inflammatory responses, and systemic alterations caused by cancer-related inflammation include an increase in both platelet and neutrophil counts as well as a decline in lymphocyte counts. Therefore, we sought to determine whether these cell counts as well as the neutrophil-to-lymphocyte ratio and the platelet-to-lymphocyte ratio in VSCC patients were also associated with disease progression, but no significant differences in those parameters between d-fVSCC and progVSCC patients were observed. 


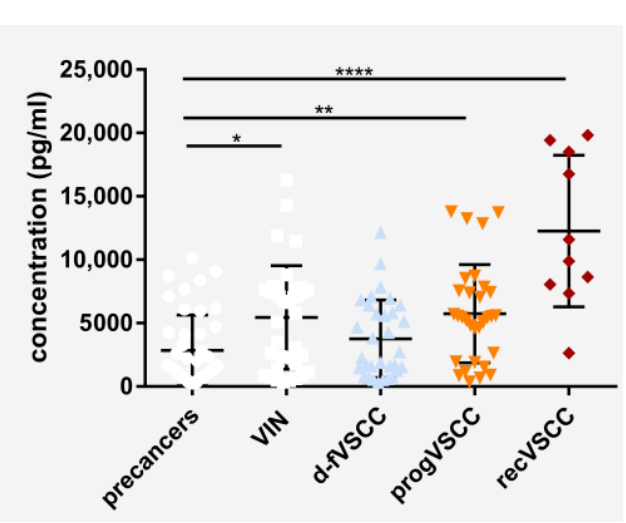

(a)

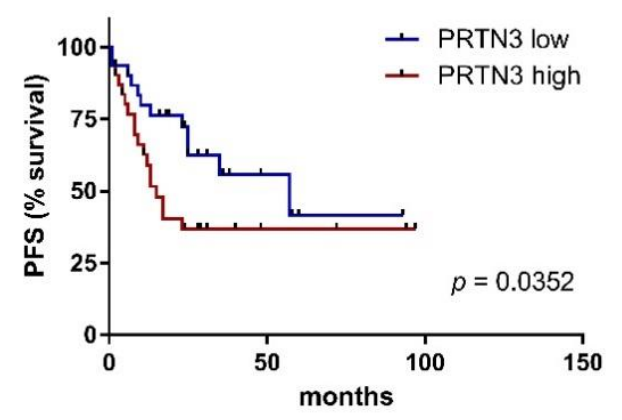

(c)

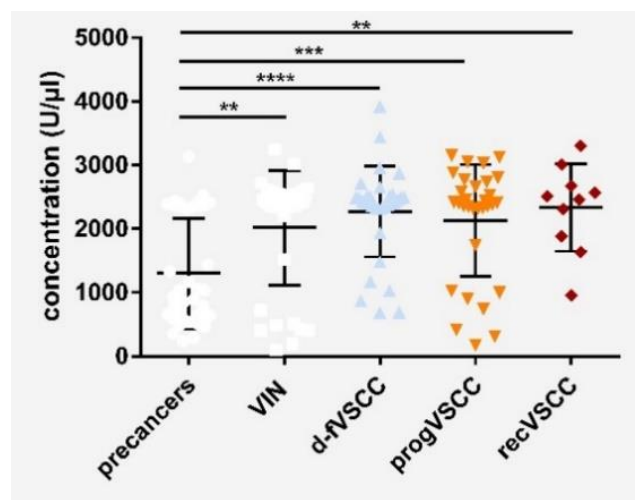

(b)

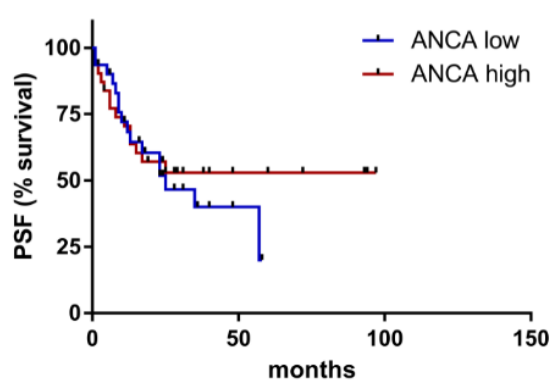

(d)

Figure 4. PRTN3 (a) and ANCA (b) serum levels. The results for the sera of 37 patients with vulvar pre-cancers (HSIL, $n=27$; dVIN, $n=7)$, patients with primary $(n=64)$ and recurrent VSCC $(n=10)$ were compared to the control sera obtained from healthy women $((\mathbf{c}), n=44)$. Survival curves (time to progression) according to the serum levels of (c) PRTN3 and ANCA in patients with primary VSCC $((\mathbf{d}), n=64)$. Significant alternations are indicated by asterisks $\left({ }^{*}, p\right.$-value $\leq 0.05 ;{ }^{* *}, p$-value $\leq 0.01$; ${ }^{* * *}, p$-value $\leq 0.001 ; * * * *, p$-value $\left.\leq 0.0001\right)$. Abbreviations: PFS, progression-free survival; high, high protein level, i.e., above the cut-off value; low-protein level, i.e., below the cut-off value (i.e., the median of protein concentration).

\subsection{The Relevance of HPV Status of VSCC Tumors}

The immune response identified as a key process driving VSCC progression could, at least partially, be explained by the consequences of HPV infection. Unexpectedly, the difference in relative numbers of HMGA2 and PTRN3-positive cells between hrHPVpositive and -negative primary VSCC samples was found to be statistically insignificant. We also compared the values of the analyzed basic hematological parameters in patients with hrHPV-positive versus hrHPV-negative VSCC tumors, and found no correlation. Similarly, no difference between PRTN3 and ANCA levels in the sera of these patients was observed.

This lack of association between these results and HPV status might be explained by the role of bacterial infections in VSCC. The Staphylococcus aureus infection pathway as second among the top KEGG pathways differentiating d-fVSCC and progVSCC proteomes (Figure S4), along with the microbicidal PRTN3 protein increase during the disease course, suggest that bacterial infections may be linked to aggressive VSCC phenotypes. Therefore, we performed PCR genotyping of 13 microorganisms known to contribute to vaginal dysbiosis on DNA isolated from 36 primary VSCC tumor samples. A pan-bacterial $16 \mathrm{~S}$ rRNA gene DNA was found in all but one sample that eventually was excluded from the analysis due to poor DNA quality. The specific bacterial species (out of 11 bacterial species analyzed) were detected in $74 \%$ of samples $(26 / 35)$ (Figure 5). The most common was E. coli $(17 / 35)$, followed by B. fragilis $(8 / 35)$, P. bivia (8/35), S. agalactiae $(8 / 35)$, S. aureus $(7 / 35)$, E. faecalis (4/35), L. iners (3/35), and L. gasseri $(2 / 35)$. 


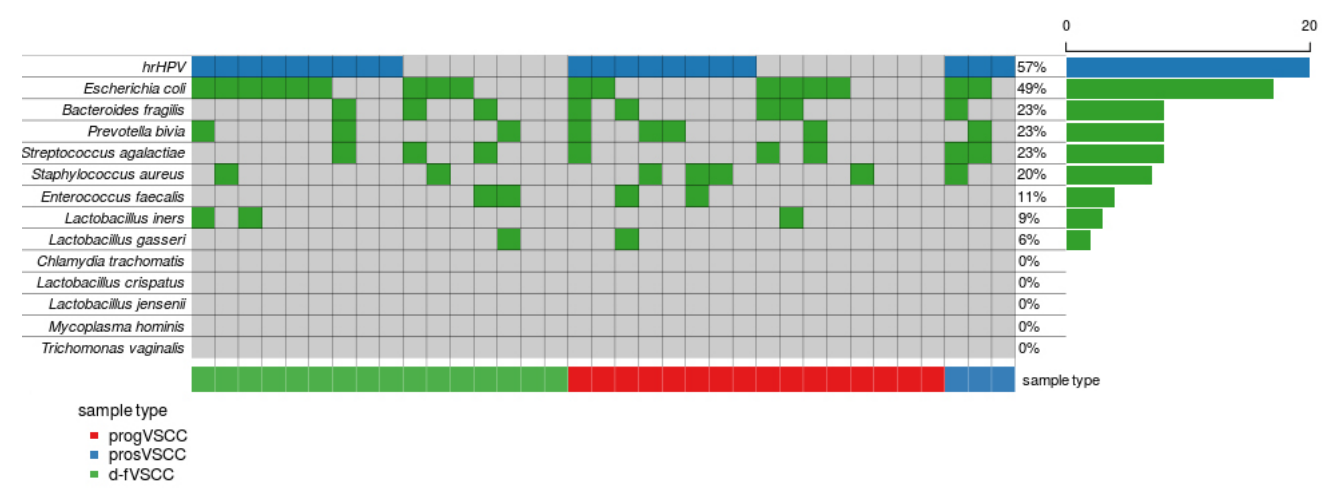

Figure 5. Distribution of bacterial species detected in VSCC samples. Each column corresponds to the individual tumor case (d-fVSCC, $n=16$; progVSCC, $n=16$ and prosVSCC, $n=3$ ). Each row corresponds to an individual microorganism with its presence or absence depicted in green or grey, respectively. The results of genotyping of 13 vaginal microorganisms is related to hrHPV status of a given tumor with the virus presence or absence depicted in blue or grey, respectively. The values on the $x$-axis describe the number (0-20) and a percentage of the samples with the presence of a given microorganism.

\section{Discussion}

Individualization of the currently available treatment modalities is hampered by the lack of prognostic factors approved for clinical practice in VSCC [16]. The experimental path that we applied, from global proteomics to the quantitative analysis of specific proteins, was aimed to advance the understanding of the mechanisms underlying VSCC progression. A similar approach was previously confirmed by Sandberg et al. [17] while detecting protein alterations related to HPV infection in a set of VSCC tumors. The authors applied iTRAQ for the analysis of 14 specimens and global data were verified by IHC. Importantly, the proteins were selected for verification based on the relapse incidence in patients and regardless of the HPV status of their tumors. A high correlation between ITRAQ and IHC results was revealed for interferon-induced GTP-binding protein Mx1 (MX1) and LGMN (Legumain). Interestingly, MX1 was also among the DEPs identified in our study. This protein was found to be over 25 -fold more abundant in VSCC compared to normal vulvar tissues according to the iTRAQ verification by PRM analysis.

In our study, we analyzed early-stage VSCC patients with a long-term follow-up after surgery. We identified the inflammatory response as the most prevalent constituent of the deregulated pathways in tumors of VSCC patients who progressed versus those of patients who were disease-free over a long follow-up time. This notion seems to be particularly justified as no immune-related pathways were found relevant when comparing VSCC tumors and normal vulvar tissues.

The immune infiltrate of VSCC and its precursor lesions by innate and adaptive immune cells, recently reviewed by Abdulrahman et al. [18], points towards the immunotherapeutic approaches as options to augment the current treatment modalities. The results of our modelling of VSCC tumorigenesis suggest that the innate immune response is a key attribute accompanying vulvar cancer progression. Local inflammatory reactions may result from a disturbed balance between the host and its commensal microbes and may promote cancer formation [19]. Neutrophil elastase (ELANE), PRTN3, and cathepsin G (CTSG) are the main neutrophil serine proteases (NSPs) released by activated neutrophils at the sites of inflammation, and are mediators of innate immune responses to microbial threats $[14,20]$. The preoperative values of neutrophil- and platelet-to-lymphocyte ratios were shown to be associated with nodal status, the most important prognostic factor in VSCC [21]. These ratios did not differ between d-fVSCC and progVSCC patients in our study, suggesting it is the activation of neutrophils and platelets during the disease course, rather than their increased numbers.

In our study, the Complement and coagulation cascades and Staphylococcus aureus infection were the two KEGG pathways the most significantly differentiating less aggressive and progressing VSCC tumors. In otherwise healthy women who contract genital tract 
infections, S. aureus is an infrequent but important pathogen [22,23]. Olejek et al. [24] found $90 \%$ of VSCC, as assessed prior to surgery, to contain pathogenic bacterial flora, including $S$. aureus species in $16 \%$ of the cases. It must be noted, however, that the $S$. aureus infection KEGG pathway may not be species-specific. Several microorganisms were previously associated with cervical cancer as well as risk for HPV infection and persistence [25]. Moreover, there is a trend toward an increased risk of cervical cancer progression in patients with vaginal dysbiosis [25]. Our preliminary PCR genotyping findings confirm the presence of pathogens, including S. aureus, within vulvar tumors.

The verification of the global protein analysis with targeted proteomics provided a list of reliable candidate biomarkers having "progression-classifier" properties, and these proteins need to be validated in future studies. In order to determine the histopathological context and cell localization of the two selected DEPs, we performed IHC analysis of VSCC tumors. HMGA2, the first verified candidate biomarker, belongs to the family of high-mobility group proteins, which alter chromatin structure and affect, either positively or negatively, the transcription of a variety of genes including genes related to the immune system functions [26,27]. HMGA2 overexpression can result from translocations at the 12 q15 region [28] or down-regulation of let-7 microRNAs [5]. In our study, we have observed decreasing levels of circulating let-7c in plasma samples from patients with vulvar premalignant lesions and VSCC. Recently, HMGA2 was demonstrated to be expressed in VSCC tumors but not in the normal vulvar tissue [29]. In our study, HMGA2 tissue abundance was associated with the aggressive phenotype of VSCC.

The second verified protein, PRTN3 (myeloblastin) belongs to a family of NSPs, which digest matrix components in inflammation and, independently of their proteolytic activity, possess microbicidal properties [12]. Bacterial infections lead to neutrophil activation triggering their antimicrobial functions, involving neutrophil phagolysosomes and neutrophil extracellular traps (NETs) that encompass multiple proteases, including PRTN3 [30]. NETs can activate the alternative complement pathway and contribute to thrombus formation while in turn, platelets stimulate neutrophils to release NET with high-mobility group box 1 (HMGB1) expression on the platelets mediating the process [31]. In our study, circulating PRTN3 and ANCA, were found to be elevated in VSCC patients as compared to healthy female donors. This observation seems to be related to neutrophil or platelet activation rather than a change in their numbers. Both local and systemic abundance of PRTN3 found in VSCC patients suggests similarities in the mechanisms underlying VSCC and development of ANCA-associated vasculitis, a group of vasculitides characterized by neutrophil-rich inflammation of small vessels and the presence of circulating ANCAs. The analogies might include a key role of bacterial infection in these conditions.

PRTN3 has been proposed as a therapeutic target for example in chronic obstructive pulmonary disease, an inflammatory condition associated with neutrophilic inflammation [32]. Sivelestat, a selective ELANE and PRTN3 inhibitor [33] was shown to improve the mortality rates of patients with sepsis associated with acute respiratory distress syndrome and disseminated intravascular coagulation [34]. ELANE and PRTN3 along with CTSG are the NSPs stored in neutrophil granules and externalized during neutrophil activation at inflammatory sites [20]. In pathological conditions, these NSPs may interact with the complement pathway causing inefficient bacterial clearance [35]. Recently, Kam et al. [36] reported an inhibition of HMGB1 with sivelestat to be able to promote myelodysplastic syndrome cell death and alter innate immune responses via suppression of NFkB pathways. As HMGA2, another high-mobility group protein, also promotes the release of pro-inflammatory cytokines through regulation of the NFKB pathway [37], one might hypothesize that sivelestat could also block HMGA2, providing an additive benefit in VSCC therapy.

\section{Patients and Methods}

4.1. Patients and Samples

Material was obtained from patients undergoing surgery for vulvar premalignant lesions and VSCC (tissue and blood samples) and from healthy volunteers (blood) at the Maria 
Sklodowska-Curie National Research Institute of Oncology in Warsaw and at the Holycross Cancer Center in Kielce, Poland, between 2002 and 2019. Patients with VSCC at early (85 FIGO stage I, 7 FIGO stage II) and advanced stages (51 FIGO stage III, 2 FIGO stage IV) as well as patients with local recurrence $(n=17)$ were enrolled. Additionally, 14 patients who underwent routine plastic surgery of the reproductive organ at the Holy Family Hospital in Warsaw, Poland, between 2014 and 2015 were assigned to the control group.

The databases of the Maria Sklodowska-Curie Institute-Oncology Center and the Holycross Cancer Center were retrospectively reviewed using the MedStream Designer application version 4.3.0.1 (Transition Technologies S.A., Poland) at the Onko.Sys platform to identify patients' clinical (including results of vulvar swabs testing for bacterial infections) and follow-up data as well as preoperative platelet, neutrophil, and lymphocyte cell counts.

The numbers of samples, according to the stages of the study, are provided in Figure 6 Primary VSCC tumors samples were divided into two categories depending of the subsequent disease course. The first comprised tumors samples of patients that were found disease-free during the follow-up period ("d-fVSCC") and the second-tumors samples obtained from patients who progressed ("progVSCC"). The progression was defined as local recurrence, regional $\mathrm{LN}$ recurrence, or cancer-related death. In addition, tumor samples obtained during vulvectomies of patients with primary VSCC enrolled prospectively (prosVSCC) and of patients with local VSCC recurrence (recVSCC) were included at the successive stages of the study. Finally, plasma samples were used for targeted protein quantitation in the blood of patients with vulvar premalignant lesions and VSCC. Normal vulvar tissue samples and sera of healthy female volunteers served as the control samples (C).
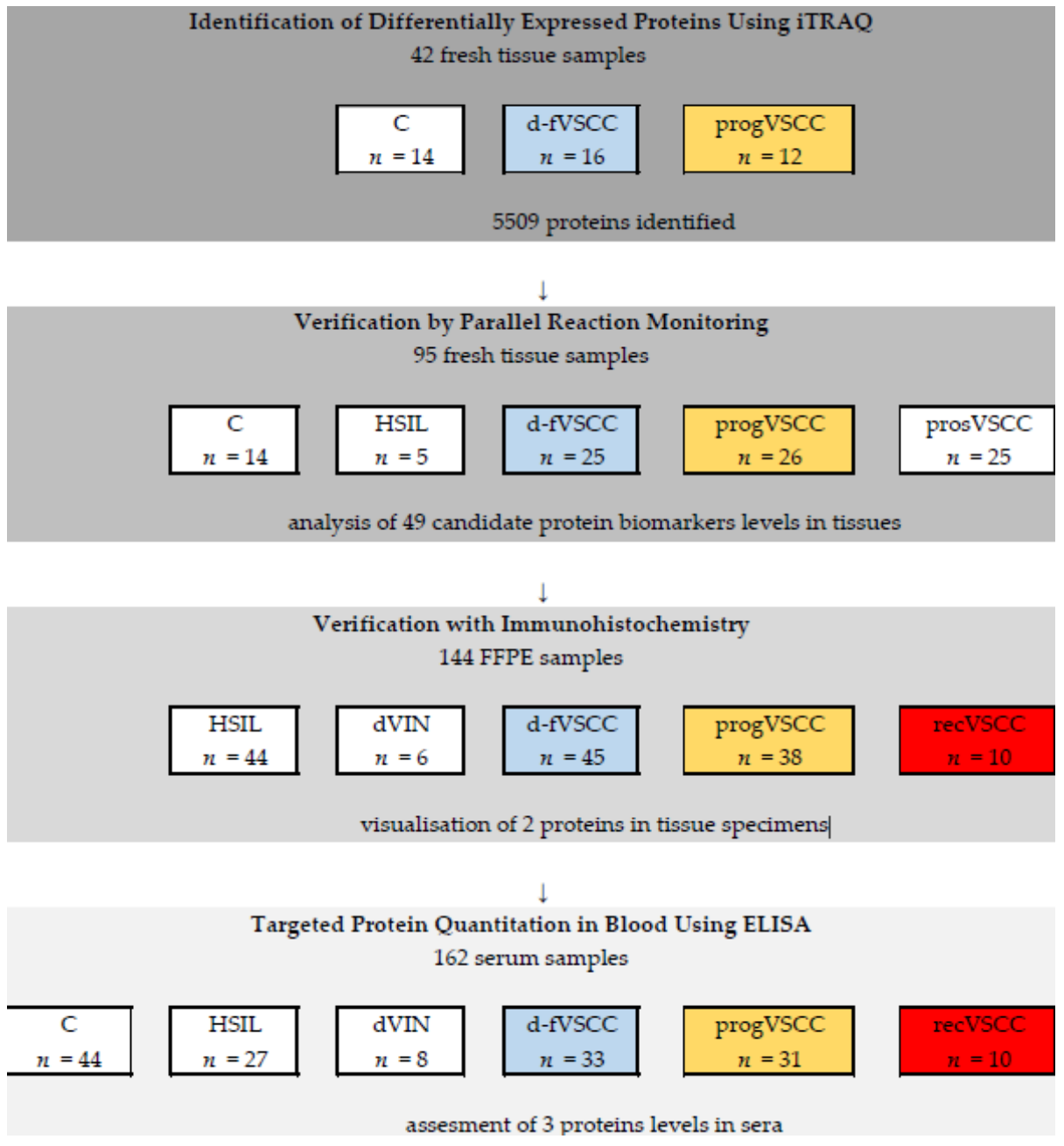

Figure 6. Study workflow from the discovery phase to protein selection and verification. 
Basic characteristics of patients with primary VSCC (i.e., those from d-fVSCC, progVSCC, and prosVSCC groups) included are shown in Table 2.

Table 2. Demographic and clinical characteristics of the patients with primary VSCC. Numbers of samples are provided for the consecutive phases of the study.

\begin{tabular}{|c|c|c|c|c|c|}
\hline \multirow{2}{*}{\multicolumn{2}{|c|}{ Characteristic }} & \multirow{4}{*}{$\begin{array}{c}\text { iTRAQ } \\
\text { Analysis } \\
70.0 \\
45.3-93.9\end{array}$} & \multicolumn{2}{|c|}{ Stage of the Study } & \multirow{4}{*}{$\begin{array}{c}\text { ELISA } \\
\text { Serum Testing } \\
72.6 \\
41.8-87.9\end{array}$} \\
\hline & & & Verification by & IHC Anglycic & \\
\hline \multirow{2}{*}{ Age-years } & Median & & 70.8 & 70.4 & \\
\hline & Range & & $45.3-94.2$ & $37.1-93.9$ & \\
\hline \multirow{4}{*}{ FIGO stage } & I & 19 & 46 & 54 & 35 \\
\hline & II & 2 & 5 & 3 & 4 \\
\hline & III & 7 & 24 & 29 & 22 \\
\hline & IV & 0 & 1 & 0 & 3 \\
\hline \multirow{4}{*}{ Histological grade } & G1 & 12 & 23 & 34 & 17 \\
\hline & G2 & 9 & 28 & 37 & 28 \\
\hline & G3 & 6 & 12 & 12 & 6 \\
\hline & $\mathrm{N} / \mathrm{D}$ & 1 & 13 & 6 & 13 \\
\hline \multirow{3}{*}{$\begin{array}{l}\text { hrHPV tumor } \\
\text { status }\end{array}$} & positive & 13 & 38 & 36 & 31 \\
\hline & negative & 15 & 27 & 32 & 6 \\
\hline & $\mathrm{N} / \mathrm{D}$ & 0 & 11 & 18 & 27 \\
\hline \multirow{3}{*}{$\begin{array}{c}\text { Disease } \\
\text { progression }\end{array}$} & d-fVSCC & 16 & 25 & 50 & 33 \\
\hline & progVSCC & 12 & 26 & 34 & 31 \\
\hline & prosVSCC & 0 & 25 & 3 & 0 \\
\hline
\end{tabular}

Abbreviations: iTRAQ, Isobaric tags for relative and absolute quantitation; PRM, parallel reaction monitoring; N/D, not determined.

\subsection{Methods}

\subsubsection{High Risk HPV Genotyping}

The hrHPV status of tissue samples was determined, as described previously [36], using the AmpliSens ${ }^{\circledR}$ HPV HCR-genotype-titre-FRT kit (InterLabService Ltd., Moscow, Russian Federation).

\subsubsection{Isobaric Tags for Relative and Absolute Quantitation (iTRAQ)}

Forty-two (14 control, 16 d-fVSCC and 12 progVSCC) samples were subjected to deep proteomic analysis using iTRAQ method. Detailed descriptions of the iTRAQ methodology and resulting data processing can be found in the Text S1 (supplementary methods). Briefly, total cell lysates were obtained from approximately $100 \mathrm{mg}$ of pulverized with the Microdismembrator II (B Braun Biotech International, Melsungen, Germany). Protein extract digestion and iTRAQ tag labeling was conducted using the 8-plex iTRAQ assay and iTRAQ Reagent and Buffer Kits (AB SCIEX, Foster City, CA, USA). A reference sample was used for normalization in each of the six independent 8-plex iTRAQ experiments.

\subsubsection{Protein Verification by Parallel Reaction Monitoring (PRM)}

To screen for the presence of candidate proteins obtained in the iTRAQ experiment, a panel of 115 peptides from 49 proteins was chosen to verify putative prognostic proteins in 25 d-fVSCC and 26 progVSCC samples. Additionally, 14 controls, 5 HSIL, and 25 prosVSCC samples were included in the PRM analysis. Plasma samples obtained from 68 patients were analyzed with PRM assays to accurately quantitate 49 qualified proteins using stableisotope-labeled internal peptide standards (3-5 peptides per protein). Detailed descriptions of the PRM methodology can be found in the Text S1.

\subsubsection{Histopathology and Immunohistochemistry}

Fifty-one formalin-fixed paraffin-embedded (FFPE) samples, consisting of 44 HSIL, six dVIN, 50 d-fVSCC, 34 progVSCC and 10 recVSCC tumor specimens, were subjected 
to IHC analysis. The diagnosis of premalignant lesions of the vulva based on histologic criteria was confirmed using anti-p16 and p53 IHC as previously described [38] and in compliance with WHO 2020 recommendations [39].

IHC staining was performed on $4 \mu \mathrm{m}$ FFPE tumor and reference tissue sections. The sections were deparaffinized with xylene and rehydrated in ethanol solutions. Heatinduced epitope retrieval was carried out in Target Retrieval Solution (Dako, Glostrup, Denmark) for $30 \mathrm{~min}$ at $96^{\circ} \mathrm{C}$. After cooling, the slides were treated for $5 \mathrm{~min}$ with an endogenous peroxidase blocker (Dako). The slides were incubated with polyclonal PRTN3 antibody (Sigma-Aldrich, Saint Louis, MO, USA; cat. No. HPA005938; 1:200 dilution) for $1 \mathrm{~h}$ at RT or with polyclonal HMGA2 antibody (GenWay Biotech. Inc, San Diego, CA, USA; cat. No. GWB-78FFEC; 1:100 dilution) at $4{ }^{\circ} \mathrm{C}$ overnight. Subsequently, the slides were labelled with the EnVision FLEX+, Mouse, High pH Detection System (Dako). The color reaction product was developed with 3,3'-diaminobenzidine tetrahydrochloride (Dako) as a substrate, and nuclear contrast was achieved with hematoxylin counterstaining. The results of immunostainings for PRTN3 and HMGA2 were assessed by counting the percentage of positive cells per 10 high power fields (HPF).

Differentiation between lymphocytes and macrophages was carried out on the basis of their morphological features such as the size and shape of the cells as wells as cytological features of the nucleus assessed at high microscopic magnification $(200-400 \times)$.

\subsubsection{Enzyme-Linked Immunosorbent Assays (ELISA)}

Sera were obtained from the blood of 35 patients with vulvar premalignant lesions (HSIL, $n=27$; dVIN, $n=8$ ), 83 VSCC patients, and 44 healthy female volunteers. Concentrations of PRTN3 protein and antibodies against PRTN3 (i.e., anti-neutrophil cytoplasmic antibodies, ANCAs) were determined by applying Human PRTN3/Myeloblastin ELISA Kit (Sandwich ELISA) (LifeSpan BioSciences, Inc., Seattle, WA, USA; cat. No. LS-F21197) and ANCA-C (PR3) (Abnova, Taiwan; cat. No. KA1084) reagents kits, respectively, according to the manufacturers' instructions. In both assays, the optical densities were read at 450 nm in Victor3 ${ }^{\mathrm{TM}}$ Plate Reader (PerkinElmer, Waltham, MA, USA).

\subsubsection{Let-7c microRNA Quantification in Plasma}

Total RNA was isolated from $200 \mu \mathrm{L}$ of plasma using miRNeasy Mini Kit (Qiagen, Hilden, Germany), and miRNA was reverse transcribed using miScript II RT Kit (Qiagen) according to the manufacturer's protocol. Expression levels of let-7c was analyzed by qRT-PCR using the miScript miRNA Arrays (Qiagen) according to the manufacturer's protocol, as described previously [40]. Hsa-let-7c expression data were normalized to two reference miRNAs, hsa-miR-93-5p and hsa-miR-425-5p.

\subsubsection{Vulvovaginal Microorganism Detection Using PCR}

Genomic DNA was isolated from 36 primary VSCC tumors (16 d-fVSCC, 17 progVSCC, and 3 prosVSCC samples) using the NucleoSpin Tissue kit (Macherey Nagel, Inc., Dueren, Germany), according to the manufacturer's protocol. Real-time PCR was performed by using an QuantStudio 5 Real-Time PCR System (Thermo Fisher Scientific, Waltham, MA, USA). The reactions were carried out in a final volume of $20 \mu \mathrm{L}$ containing $10 \mu \mathrm{L}$ TaqMan $^{\mathrm{TM}}$ Gene Expression Master Mix (Thermo Fisher Scientific) and $5 \mathrm{ng}$ of template DNA. The reaction was performed in TaqMan Array 96 Plates (Thermo Fisher Scientific) containing primers and probes for 11 bacterial species, Mycoplasma hominis and Trichomonas vaginalis (listed in Supplementary Figure S3). The thermal cycling conditions were: $95^{\circ} \mathrm{C}$ for $10 \mathrm{~min}$, 45 cycles of 15 seconds at $95^{\circ} \mathrm{C}$, and $1 \mathrm{~min}$ at $60^{\circ} \mathrm{C}$. The microorganisms' genotyping results were visualized as Oncoplots plotted using Maftools from R Bioconductor package [41].

\subsubsection{Statistical Analyses}

iTRAQ data analysis was performed using the MScan, MSparky, MStat, and Diffprot software tools [42]. Differentially expressed proteins (DEPs) in d-fVSCC and progVSCC 
samples were searched (both for iTRAQ and PRM) by Wilcoxon test, with the statistical significance threshold set at $p$-value $<0.05$. The Benjamini-Hochberg procedure was used to adjust $p$-values due to multiple hypothesis testing. Overrepresentation of Gene Ontology (GO) terms [43] and Kyoto Encyclopedia of Genes and Genomes (KEGG) pathways [44] was conducted with GOstats version 2.42 [45], GO.db version 3.4.1, org.Hs.eg.db version 3.4.1, annotate version 1.54.0, and AnnotationDbi version 1.38.2 packages. For each GO/KEGG database, overrepresentation of the respective categories was tested among the top $10 \%$ of proteins, according to $p$-value. Areas under receiver operating characteristics (ROC) curves (AUC) used to assess the performance of DEPs in PRM analysis, along with GO and KEGG overrepresentation, were calculated in the R environment version 3.4.1 (http:/ / www.R-project.org). For canonical pathways (and network analyses) DEPs were submitted to Ingenuity Pathway Analysis (Ingenuity Systems, USA; http:/ / www.ingenuity.com, accessed on 9 August 2019).

The Kruskal-Wallis test followed by Wilcoxon multiple pair-wise comparisons was used to test the significance of differences in protein expression between the tissue and serum sample groups. The Benjamini-Hochberg procedure was used to adjust $p$-values due to multiple hypothesis testing. $p$-value $<0.05$ was considered significant. Median follow-up time of the enrolled patient was determined from the date of surgery to the date of death or the date of the last interview was registered. Association between the protein content of selected proteins in both tumor and blood samples with time to progression of the disease in patients with primary VSCC was determined using Cox proportional hazard model implemented in the survival package (version 2.41-3) in the R environment.

The results were visualized using GraphPadPrism (San Diego, CA, USA).

\section{Conclusions}

In summary, the biological insight from the study points at inflammation as a key event during VSCC tumorigenesis. Indirect but numerous lines of evidence suggest that changes in the genital microbial flora disturbances may be the causative agents in vulvar cancer-related inflammation. Although vulvar carcinoma does not present a diagnostic challenge, methods to predict the disease course in early-stage VSCC patients are missing. In early-stage VSCC, HMGA2 and PRTN3 should be considered as potential predictive markers and putative therapeutic targets. Increased abundance of HMGA2 and PRTN3 in aggressive VSCC was found to be independent of the hrHPV status of the tumors. This further suggests that bacterial rather than viral infection status should be considered in the development of targeted therapies in VSCC. Prevention and control of bacterial infections are anticipated to become a valuable addition to current cancer treatment modalities $[46,47]$. Finally, several similarities in the biology of AVV provides grounds for the assumption that VSCC patients might benefit from treatments currently applied for AVV and other neutrophilic diseases.

Supplementary Materials: The following are available online at https: / /www.mdpi.com/2072-6 694/13/1/27/s1, Text S1: Supplementary Methods, Figure S1: Overlapping canonical pathways identified by core analysis in Ingenuity Pathway Analysis (IPA) that were significantly enriched in the dataset, Figure S2: Let-7c levels in plasma samples of patients with VIN $(n=21)$, primary VSCC $(n=34)$ and recurrent VSCC (recVSCC, $n=2)$ as compared to control plasma samples obtained from healthy women (C, $n=15)$, Figure S3: KEGG pathway view on proteomic data (hsa04610), Figure S4: Staphylococcus aureus infection (hsa5150) KEGG pathway, Table S1: The top 20 overrepresented GO term for DEPs in VSCC and normal vulvar tissues, Table S2. The top 20 KEGG pathways in VSCC and normal vulvar tissues, Table S3: The top 20 overrepresented GO terms for DEPs in progVSCC versus d-fVSCC samples, Table S4: The top 20 KEGG pathways for DEPs overrepresented in progVSCC versus d-fVSCC samples, Table S5: Candidate VSCC biomarkers examined in PRM analysis.

Author Contributions: Proteomic experiments, A.F.; design and proteomic experiments, D.D.; verification experiments (immunohistochemical and ELISA analyses), N.R.; histological evaluation of the samples, E.B.-Z.; clinical specimen collection and interpretation of the clinical results, A.K., A.W., and K.Z.; microorganism detection and data analysis, S.Z.; data analysis, K.G. and M.K.; conceptualization 
and funding acquisition, M.K.; supervision, M.K.; project administration, M.K.; writing-original draft preparation, M.K.; manuscript drafting and revision, A.K.; writing-review and editing, all authors. All authors have read and agreed to the published version of the manuscript.

Funding: This research was funded by the Polish National Science Centre, grant No. 2013/10/E/NZ5/ 00663. The used proteomics equipment of the Mass Spectrometry Laboratory was sponsored in part by the Centre for Preclinical Research and Technology (CePT), a project co-sponsored by European Regional Development Fund and Innovative Economy, The National Cohesion Strategy of Poland (POIG.02.02.00-14-024/08-00).

Institutional Review Board Statement: The study received ethical institutional board approvals of the Maria Sklodowska-Curie Institute-Oncology Center (No. 44/2002, 16/2015), Holycross Cancer Center (No. 15/2014) and Holy Family Hospital (2014-11-26).

Informed Consent Statement: Not applicable.

Data Availability Statement: Proteomic data is uploaded to a public proteomic repository and will be shared upon request to the corresponding author.

Acknowledgments: We would like to thank Jacek Olędzki from the IBB MS Laboratory for his valuable technical assistance.

Conflicts of Interest: The authors declare no conflict of interest.

\section{References}

1. Bray, F.; Ferlay, J.; Soerjomataram, I.; Siegel, R.L.; Torre, L.A.; Jemal, A. Global cancer statistics 2018: GLOBOCAN estimates of incidence and mortality worldwide for 36 cancers in 185 countries. CA Cancer J. Clin. 2018, 68, 394-424. [CrossRef]

2. Cancer Stat Facts: Vulvar Cancer. Available online: https://seer.cancer.gov/statfacts/html/vulva.html (accessed on 5 December 2020).

3. The European Society of Gynaecologic Oncology (ESGO) Vulvar Cancer Guidelines. Available online: https: / guidelines.esgo. org/media/2016/08/ESGO-Vulvar-cancer-Complete-report-fxd2.pdf (accessed on 5 December 2020).

4. Gill, B.S.; Bernard, M.E.; Lin, J.F.; Balasubramani, G.K.; Rajagopalan, M.S.; Sukumvanich, P.; Krivak, T.C.; Olawaiye, A.B.; Kelley, J.L.; Beriwal, S. Impact of adjuvant chemotherapy with radiation for node-positive vulvar cancer: A National Cancer Data Base (NCDB) analysis. Gynecol. Oncol. 2015, 137, 365-372. [CrossRef]

5. Gadducci, A.; Aletti, G.D. Locally advanced squamous cell carcinoma of the vulva: A challenging question for gynecologic oncologists. Gynecol. Oncol. 2020, 158, 208-217. [CrossRef]

6. Cohen, P.A.; Anderson, L.; Eva, L.; Scurry, J. Clinical and molecular classification of vulvar squamous pre-cancers. Int. J. Gynecol. Cancer 2019, 29, 821-828. [CrossRef] [PubMed]

7. Horne, Z.D.; Dohopolski, M.J.; Pradhan, D.; Bhargava, R.; Edwards, R.P.; Kelley, J.L.; Comerci, J.T.; Olawaiye, A.B.; CourtneyBrooks, M.B.; Bockmeier, M.M.; et al. Human papillomavirus infection mediates response and outcome of vulvar squamous cell carcinomas treated with radiation therapy. Gynecol. Oncol. 2018, 151, 96-101. [CrossRef] [PubMed]

8. Clancy, A.A.; Spaans, J.N.; Weberpals, J.I. The forgotten woman's cancer: Vulvar squamous cell carcinoma (VSCC) and a targeted approach to therapy. Ann. Oncol. 2016, 27, 1696-1705. [CrossRef] [PubMed]

9. Zieba, S.; Chechlinska, M.; Kowalik, A.; Kowalewska, M. Genes, pathways and vulvar carcinoma-New insights from nextgeneration sequencing studies. Gynecol. Oncol. 2020, 158, 498-506. [CrossRef]

10. Belczacka, I.; Latosinska, A.; Metzger, J.; Marx, D.; Vlahou, A.; Mischak, H.; Frantzi, M. Proteomics biomarkers for solid tumors: Current status and future prospects. Mass Spectrom Rev. 2019, 38, 49-78. [CrossRef]

11. Balzeau, J.; Menezes, M.R.; Cao, S.; Hagan, J.P. The LIN28/let-7 Pathway in Cancer. Front. Genet. 2017, 8, 31. [CrossRef]

12. Skold, S.; Rosberg, B.; Gullberg, U.; Olofsson, T. A secreted proform of neutrophil proteinase 3 regulates the proliferation of granulopoietic progenitor cells. Blood 1999, 93, 849-856. [CrossRef]

13. von Vietinghoff, S.; Eulenberg, C.; Wellner, M.; Luft, F.C.; Kettritz, R. Neutrophil surface presentation of the anti-neutrophil cytoplasmic antibody-antigen proteinase 3 depends on N-terminal processing. Clin. Exp. Immunol. 2008, 152, 508-516. [CrossRef] [PubMed]

14. Meyer-Hoffert, U.; Wiedow, O. Neutrophil serine proteases: Mediators of innate immune responses. Curr. Opin. Hematol. 2011, 18, 19-24. [CrossRef] [PubMed]

15. Peng, X.; Ramstrom, S.; Kurz, T.; Grenegard, M.; Segelmark, M. The neutrophil serine protease PR3 induces shape change of platelets via the Rho/Rho kinase and $\mathrm{Ca}\left({ }^{2+}\right)$ signaling pathways. Thromb. Res. 2014, 134, 418-425. [CrossRef] [PubMed]

16. Mitra, S.; Sharma, M.K.; Kaur, I.; Khurana, R.; Modi, K.B.; Narang, R.; Mandal, A.; Dutta, S. Vulvar carcinoma: Dilemma, debates, and decisions. Cancer Manag. Res. 2018, 10, 61-68. [CrossRef]

17. Sandberg, A.; Lindell, G.; Kallstrom, B.N.; Branca, R.M.; Danielsson, K.G.; Dahlberg, M.; Larson, B.; Forshed, J.; Lehtio, J. Tumor proteomics by multivariate analysis on individual pathway data for characterization of vulvar cancer phenotypes. Mol. Cell Proteomics 2012, 11, M112.016998. [CrossRef] 
18. Abdulrahman, Z.; Kortekaas, K.E.; De Vos Van Steenwijk, P.J.; Van Der Burg, S.H.; Van Poelgeest, M.I. The immune microenvironment in vulvar (pre)cancer: Review of literature and implications for immunotherapy. Expert Opin. Biol. Ther. 2018, 18, 1223-1233. [CrossRef]

19. Robertson, E.S. Microbiome and Cancer; Humana Press: Cham, Switzerland, 2019. [CrossRef]

20. Korkmaz, B.; Horwitz, M.S.; Jenne, D.E.; Gauthier, F. Neutrophil elastase, proteinase 3, and cathepsin G as therapeutic targets in human diseases. Pharmacol. Rev. 2010, 62, 726-759. [CrossRef]

21. Ertas, I.E.; Gungorduk, K.; Akman, L.; Ozdemir, A.; Terek, M.C.; Ozsaran, A.; Sanci, M.; Dikmen, Y. Can preoperative neutrophil:lymphocyte and platelet:lymphocyte ratios be used as predictive markers for lymph node metastasis in squamous cell carcinoma of the vulva? Eur. J. Obstet. Gynecol. Reprod. Biol. 2013, 171, 138-142. [CrossRef]

22. Gibbs, R.S. Microbiology of the female genital tract. Am. J. Obstet. Gynecol. 1987, 156, 491-495. [CrossRef]

23. Singh, J.; Kalia, N.; Kaur, M. Recurrent Vulvovaginal Infections: Etiology, Diagnosis, Treatment and Management. In Infectious Diseases and Your Health.; Singh, P.P., Ed.; Springer: Singapore, 2018.

24. Olejek, A.; Kozak-Darmas, I.; Kellas-Sleczka, S.; Nowak, L. The usefulness of bacteriological screening in wound healing aspect after vulvectomy. Menopause Rev. 2005, 4, 33-36.

25. Laniewski, P.; Ilhan, Z.E.; Herbst-Kralovetz, M.M. The microbiome and gynaecological cancer development, prevention and therapy. Nat. Rev. Urol. 2020, 17, 232-250. [CrossRef] [PubMed]

26. Cleynen, I.; Van de Ven, W.J. The HMGA proteins: A myriad of functions (Review). Int. J. Oncol. 2008, 32, 289-305. [CrossRef] [PubMed]

27. Reeves, R.; Beckerbauer, L. HMGI/Y proteins: Flexible regulators of transcription and chromatin structure. Biochim. Biophys. Acta 2001, 1519, 13-29. [CrossRef]

28. Fusco, A.; Fedele, M. Roles of HMGA proteins in cancer. Nat. Rev. Cancer 2007, 7, 899-910. [CrossRef]

29. Agostini, A.; Panagopoulos, I.; Andersen, H.K.; Johannesen, L.E.; Davidson, B.; Trope, C.G.; Heim, S.; Micci, F. HMGA2 expression pattern and TERT mutations in tumors of the vulva. Oncol. Rep. 2015, 33, 2675-2680. [CrossRef]

30. Clancy, D.M.; Henry, C.M.; Sullivan, G.P.; Martin, S.J. Neutrophil extracellular traps can serve as platforms for processing and activation of IL-1 family cytokines. FEBS J. 2017, 284, 1712-1725. [CrossRef]

31. Soderberg, D.; Segelmark, M. Neutrophil Extracellular Traps in ANCA-Associated Vasculitis. Front. Immunol. 2016, 7, 256. [CrossRef]

32. Crisford, H.; Sapey, E.; Stockley, R.A. Proteinase 3; a potential target in chronic obstructive pulmonary disease and other chronic inflammatory diseases. Respir. Res. 2018, 19, 180. [CrossRef]

33. Pharos database entry. Available online: https://pharos.nih.gov/idg/ligands/sivelestat (accessed on 9 October 2019).

34. Hayakawa, M.; Katabami, K.; Wada, T.; Sugano, M.; Hoshino, H.; Sawamura, A.; Gando, S. Sivelestat (selective neutrophil elastase inhibitor) improves the mortality rate of sepsis associated with both acute respiratory distress syndrome and disseminated intravascular coagulation patients. Shock 2010, 33, 14-18. [CrossRef]

35. van den Berg, C.W.; Tambourgi, D.V.; Clark, H.W.; Hoong, S.J.; Spiller, O.B.; McGreal, E.P. Mechanism of neutrophil dysfunction: Neutrophil serine proteases cleave and inactivate the C5a receptor. J. Immunol. 2014, 192, 1787-1795. [CrossRef]

36. Kam, A.Y.F.; Piryani, S.O.; McCall, C.M.; Park, H.S.; Rizzieri, D.A.; Doan, P.L. Targeting High Mobility Group Box-1 (HMGB1) Promotes Cell Death in Myelodysplastic Syndrome. Clin. Cancer Res. 2019, 25, 4155-4167. [CrossRef] [PubMed]

37. Huang, H.; Li, H.; Chen, X.; Yang, Y.; Li, X.; Li, W.; Huang, C.; Meng, X.; Zhang, L.; Li, J. HMGA2, a driver of inflammation, is associated with hypermethylation in acute liver injury. Toxicol. Appl. Pharmacol. 2017, 328, 34-45. [CrossRef] [PubMed]

38. Zieba, S.; Kowalik, A.; Zalewski, K.; Rusetska, N.; Goryca, K.; Piascik, A.; Misiek, M.; Bakula-Zalewska, E.; Kopczynski, J.; Kowalski, K.; et al. Somatic mutation profiling of vulvar cancer: Exploring therapeutic targets. Gynecol. Oncol. 2018, 150, 552-561. [CrossRef]

39. WHO Classification of Tumours. Available online: https://tumourclassification.iarc.who.int/welcome/ (accessed on 8 December 2020).

40. Zalewski, K.; Misiek, M.; Kowalik, A.; Bakula-Zalewska, E.; Kopczynski, J.; Zielinska, A.; Bidzinski, M.; Radziszewski, J.; Gozdz, S.; Kowalewska, M. Normalizers for microRNA quantification in plasma of patients with vulvar intraepithelial neoplasia lesions and vulvar carcinoma. Tumour Biol. 2017, 39, 1010428317717140. [CrossRef] [PubMed]

41. Mayakonda, A.; Lin, D.C.; Assenov, Y.; Plass, C.; Koeffler, H.P. Maftools: Efficient and comprehensive analysis of somatic variants in cancer. Genome Res. 2018, 28, 1747-1756. [CrossRef]

42. Malinowska, A.; Kistowski, M.; Bakun, M.; Rubel, T.; Tkaczyk, M.; Mierzejewska, J.; Dadlez, M. Diffprot-software for nonparametric statistical analysis of differential proteomics data. J. Proteomics 2012, 75, 4062-4073. [CrossRef]

43. The Gene Ontology, C. The Gene Ontology Resource: 20 years and still GOing strong. Nucleic Acids Res. 2019, 47, D330-D338. [CrossRef]

44. Kanehisa, M. Toward understanding the origin and evolution of cellular organisms. Protein Sci. 2019, 28, 1947-1951. [CrossRef]

45. Falcon, S.; Gentleman, R. Using GOstats to test gene lists for GO term association. Bioinformatics 2007, 23, 257-258. [CrossRef]

46. van Elsland, D.; Neefjes, J. Bacterial infections and cancer. EMBO Rep. 2018, 19. [CrossRef]

47. Picardo, S.L.; Coburn, B.; Hansen, A.R. The microbiome and cancer for clinicians. Crit. Rev. Oncol. Hematol. 2019, 141, 1-12. [CrossRef] [PubMed] 